\title{
Comprender la pobreza: debate entre John Rawls y Amartya Sen
}

\author{
Understanding poverty: \\ A debate between John Rawls and Amartya Sen
}

\author{
EGUZKI URTEAGA \\ Universidad del País Vasco (España)
}

Recibido: 07-05-2010 Aprobado definitivamente: 30-05-2011

\section{RESUMEN}

Reconstrucción teórica de nuestras intuiciones a propósito de la justicia social, la Teoría de la justicia de John Rawls debía tomar en consideración a los más desfavorecidos a través del principio de diferencia que reparte los bienes de manera equitativa. Para Rawls, la objetividad moral está garantizada por la experiencia del posicionamiento original, caracterizada por el velo de ignorancia. Amartya Sen cuestiona ese planteamiento criticando el índice de los bienes básicos. Este artículo explicita lo que está en juego en este debate y desarrolla las aportaciones de la teoría de las capacidades a la reflexión contemporánea sobre las desigualdades socioeconómicas.

$$
\begin{gathered}
\text { PALABRAS CLAVE } \\
\text { RAWLS - SEN - TEORÍA - POBREZA - CAPACIDAD }
\end{gathered}
$$

\footnotetext{
ABSTRACT

In his theoretical reconstruction of our intuitions about social justice, John Rawls's Theory of justice intended to take into consideration the worst-off due to the difference principle that distributes the goods in an equal way. For Rawls, the moral objectivity is guaranteed by the
}

(C) Contrastes. Revista Internacional de Filosofía, vol. XVII (2012), pp. 269-286. ISSN: 1136-4076

Departamento de Filosofía, Universidad de Málaga, Facultad de Filosofía y Letras

Campus de Teatinos, E-29071 Málaga (España) 
experience of the original position, characterized by the veil of ignorance that conceals particular interests. But, Amartya Sen has criticized this theory and specially the index of basic goods. This article states explicitly what is at stake in this debate and develops the contributions of the theory of capabilities to the contemporary reflection on the economic and social inequalities.

KEYWORDS

RAWLS - SEN - THEORY - POVERTY - CAPABILITY

\section{INTRODUCCIÓN}

La eXIGENCIA DE JUSTiCia es también una demanda de igualdad. Pero, ¿de qué igualdad se trata? Los fenómenos de pobreza nos conducen a reflexionar sobre la noción de justicia; la aplicación de las leyes y reglamentos en vigor no es siempre suficiente para garantizar la equidad, y es por esta razón que la filosofía siente la necesidad de repensar las condiciones de legitimidad de estas leyes y reglamentos. No obstante, los problemas vinculados a la pobreza requieren unas soluciones eficaces, y este aspecto práctico del problema se añade a la preocupación por la legitimidad. Entre los escritos fundamentales de la filosofía política contemporánea, la Teoría de la justicia de John Rawls (1971) ha convertido la justicia en el criterio determinante de las instituciones en una sociedad bien ordenada: «la justicia es la primera virtud de las instituciones sociales así como la verdad es la de los sistemas de pensamiento» (Rawls 1971, p. 29).

La analogía entre verdad y justicia convierte a Rawls en un defensor de la posibilidad de un acuerdo universal entre los individuos a propósito de lo que es la justicia. Si las concepciones de la justicia pueden divergir, el concepto de justicia es inalterable. Asegura la adecuación de los principios a la sociedad, concebida como una comunidad regida por unas instituciones. La sociedad bien ordenada es una sociedad regida por dos principios de justicia concebida como equidad: el principio de libertad igual para todos y el segundo principio, que se refiere a la igualdad de oportunidades y al principio de diferencia, según el cual las desigualdades económicas y sociales son justas si aventajan a los más vulnerables. Este principio de diferencia implica una justificación de las desigualdades económicas y sociales en el seno de una sociedad justa.

La concepción ética de la justicia social se fundamenta, por lo tanto, en la definición de la pobreza que presupone. Pero, la elección de un método descriptivo de los fenómenos de pobreza determina igualmente la puesta en marcha de una estrategia de lucha contra esta última definiendo los fundamentos éticos de las instituciones de regulación y de ayuda. Los trabajos de Amartya Sen sobre los mecanismos de la pobreza y sobre las cuestiones vinculadas al 
desarrollo económico, han contribuido a afinar la concepción de la pobreza de los economistas y de las instituciones internacionales. Sen recuerda sistemáticamente que Rawls ha insistido en la importancia de defender las libertades políticas y de tratar las desigualdades económicas y sociales que permiten a los más desprovistos ejercer planamente sus derechos. No obstante, ha criticado el índice de los «bienes primeros» o «bienes básicos», índice creado por Rawls con el fin de definir las necesidades mínimas de los ciudadanos en una sociedad justa, tanto en términos de libertades como de recursos. Sen ha opuesto a este índice la noción de «capacidad», que toma en consideración las variaciones interindividuales.

A esta crítica, Rawls ha contestado en dos tiempos, y, aunque no cuestione explícitamente su primera respuesta, ésta difiere notablemente de la segunda. Efectivamente, la primera respuesta ha situado la crítica de Sen a nivel de la distinción entre el ámbito político y el ámbito de las doctrinas comprensivas tales como las religiones. La respuesta siguiente se ha interesado por las variaciones de las capacidades a propiamente dichas según los individuos. Pero, sean cual sean las concesiones que Rawls hace a Sen, rechaza cuestionar las condiciones de legitimidad de la justicia.

La condición de legitimidad de los principios de justicia está garantizada por el posicionamiento original en el cual se encuentran los socios que construyen estos principios. Esta experiencia cognitiva permite hacer resaltar las motivaciones morales de estos socios concebidos como unos «agentes políticos puros», es decir liberados de unas motivaciones ajenas a la preocupación de la justicia como equidad. Esta condición implica una restricción de la base de información disponible para elaborar estos principios y definir la pobreza. No en vano, ante las múltiples formas de la pobreza, podemos preguntarnos sobre la pertinencia del término de pobreza. La identificación de las personas con desventaja a nivel del posicionamiento original, es decir de manera abstracta, pone en peligro la legitimidad de la teoría de la justicia a dos niveles: por una parte, la utilización de criterios de definición de los colectivos con más desventaja no se derivan exclusivamente de unas motivaciones aceptables, y, por otra parte, el hecho de descuidar las consecuencias de una caracterización demasiado abstracta de la categoría de pobre puede alejar la teoría de su objetivo de equidad. Por lo tanto, el nivel de abstracción requerido para la legitimación de unos principios de justicia parece difícilmente compatible con las realidades cambiantes que caracterizan los fenómenos de pobreza. ¿Pero una caracterización demasiado cambiante de los fenómenos de pobreza no es arbitraria?

Tanto Rawls como Sen han intentado evitar la parte de arbitrariedad moral contenida en la elección del criterio de definición del grupo más desfavorecido. Rawls ha procedido por abstracción, mientras que Sen ha intentado hacer coexistir deontología y «consecuencialismo» a través de la noción de 
libertad instrumental. Para ello, ha privilegiado la descripción como condición previa para la definición de la pobreza. Pero, ¿en nombre de qué un fenómeno de pobreza descrito por un economista sería injusto si los criterios de justicia están invalidados por esta descripción? La crítica de Sen se sitúa a nivel de la descripción de la categoría de los que tienen más desventaja, mientras que la teoría de a justicia reviste una dimensión prescriptiva, como ideal regulador para una sociedad política. ¿Esta crítica es pertinente? ¿Cuáles son las interacciones entre descripción y prescripción tanto en la teoría de la justicia como en el enfoque de las capacidades defendida por Sen? En resumidas cuentas, ¿es posible establecer un criterio de definición de la pobreza que excluya cualquier forma de arbitrariedad? ¿Se puede reconocer y definir la pobreza de manera universal y necesaria, y así aplicar el principio de diferencia de John Rawls?

\section{DefiniR LAS PERSONAS CON DESVENTAJA EN UNA TEORÍA DE LA JUSTICIA COMO EQUIDAD}

La dimensión originaria del contrato propuesto en la Teoría de la justicia se traduce por unas «ideas intuitivas fundamentales», verdaderos requisitos previos de la teoría, a partir de los cuales cualquier actor racional puede establecer un acuerdo. La universalidad de estas ideas resulta de la concepción kantiana de la razón práctica: estas ideas son análogas a las propuestas fundamentales de la razón práctica pura (Kant 2000). Dichas ideas son las siguientes:

Para los socios que establecen la convención originaria, la justicia es el criterio determinante de la validez de las instituciones políticas y sociales en una sociedad dada.

La estructura básica de la sociedad es el propio objeto de la teoría de la justicia; la estructura básica puede ser definida como la manera según la cual las instituciones políticas y sociales determinan el carácter más o menos favorable de los posicionamientos sociales de cada uno.

Los principios de justicia son objeto de un acuerdo original establecido en un posicionamiento inicial de igualdad de todos los socios; los conocimientos de estos socios son limitados por un «velo de ignorancia», que excluye cualquier conocimiento particular de su posicionamiento social real. Esta limitación es la condición que posibilita una elección desinteresada.

\section{II.1. UN ENFOQUE HOLÍSTICO QUE NO SEPARA LA LIBERTAD Y LA IGUALDAD}

La perspectiva de Rawls implica una atención particular hacia las libertades así como una cierta forma de justicia redistributiva. La teoría de la justicia determina la organización social: «la estructura básica de la sociedad es el primer objeto de la justicia puesto que sus efectos son muy profundos y se hacen sentir 
desde el inicio» (Rawls 1971, p. 33). La existencia de desigualdades sociales confiere su sentido a la teoría de la justicia como equidad. Los socios que ponen en marcha el acuerdo original tienen un conocimiento general del reparto de las ventajas de una sociedad. Dos factores de desigualdad son puestos de manifiesto: el «sistema político» del que dependen las desigualdades de tipo político o jurídico, y las circunstancias socioeconómicas, que determinan las desigualdades económicas y sociales. A partir de entonces podemos preguntarnos si Rawls tratará estas desigualdades conjuntamente o separadamente. «Las instituciones sociales favorecen ciertos puntos de partida en detrimento de otros. Se trata de desigualdades especialmente profundas».

Las desigualdades de la sociedad real están en contradicción con el posicionamiento de igualdad ideal que es la del ciudadano como tal, es decir como actor político puro en el seno de un sistema democrático, y no como miembro de la sociedad civil. Las nociones de «mérito» y de «valor» no pueden ser tomados en consideración a la hora de conceder la ciudadanía. Plantean unos problemas en cuanto a su toma en consideración en el reparto de las ventajas. Se espera de una teoría de la justicia el tratamiento del vínculo entre igualdad política y desigualdades económicas. Dicho de otra forma, ¿en qué medida la esfera política engloba las cuestiones de orden económico y social? «Por lo tanto, es a estas desigualdades, probablemente inevitables en la estructura básica de cualquier sociedad, que los principios de justicia social deben aplicarse en primer lugar».

La teoría de la justicia no niega una cierta independencia de las desigualdades con respeto a las instituciones: estas constituyen un hecho, al que el derecho debe aportar las correcciones necesarias para su buen funcionamiento. La hipótesis del posicionamiento original permite a John Rawls hacer resaltar dos principios de justicia. Estos principios que, en virtud de las condiciones del posicionamiento original, estarían elegidos por una asociación de personas libres, iguales, racionales y desinteresadas, y, por lo tanto, deseosas de favorecer los intereses superiores de su razón. Estos dos principios de justicia están vinculados entre sí. Su formulación evoluciona a lo largo de la Teoría de la justicia, y, en su último libro fundamental titulado Liberalismo político, Rawls propone una nueva versión de esta teoría.

En la formulación definitiva de la Teoría de la justicia, el primer principio reconoce a cada persona un «derecho comparable a disfrutar del sistema más extendido de libertades básicas iguales para todos, compatible con un sistema para todos» (Rawls 1971, p. 341). Este principio estipula que unas instituciones justas reconocen a cada ciudadano la posibilidad de gozar de libertades fundamentales en el límite del disfrute de sus propias libertades por otro ciudadano. El segundo principio plantea unas condiciones a las desigualdades económicas y sociales. Está subdividido en un principio de igualdad de oportunidades que 
limita la justificación de las desigualdades a las que están «vinculadas a unas posiciones y funciones abiertas para todos», $\mathrm{y}$ en un principio de diferencia que solo justifica aquellas que benefician al mayor número de personas con desventaja, en el límite de un justo principio del ahorro (Rawls 1971, p. 341).

Posteriormente, Rawls enuncia dos prioridades, la prioridad de la libertad sobre el segundo principio, ya que la libertad solo puede ser limitada en nombre de la libertad, y la prioridad de los principios de justicia sobre los principios de eficacia y bienestar, tal como el principio utilitarista de la maximización de la suma total de ventajas.

Las libertades básicas iguales para todos son las siguientes: las libertades políticas (derecho de voto y de elegibilidad, etc.), la libertad de expresión y de reunión, la libertad de conciencia, la integridad de la persona, el derecho de propiedad personal y el acceso a una justicia que corresponda a los principios del Estado de derecho (Rawls 1971,p. 92). Ese principio es seguido por un principio de igualdad de oportunidades, así como por un «principio de diferencia» y un principio de justificación de las desigualdades que aventajan a los que tienen más desventaja. Pero, el hecho de que la estructura básica sea objeto de una teoría de la justicia como equidad implica quizá que este tipo de desigualdades sea inexistente o sea poco pertinente para una reflexión, a la imagen de la que lleva a cabo Rawls. No en vano, la ambigüedad persiste a ese nivel de reflexión. Efectivamente, la primera formulación del principio de diferencia preconiza que las desigualdades beneficien a cada uno, que sea ventajado o no. En este sentido, el criterio de desventaja solo parece llegar en un segundo momento, con el fin de hacer el reparto más eficaz en términos de justicia social.

El primer principio es prioritario con respecto al segundo, lo que significa que, en una sociedad bien ordenada, las libertades no pueden ser sacrificadas, parcialmente o totalmente, a un bienestar superior o para reforzar la igualdad de oportunidades. El sacrificio de una libertad particular solo puede estar justificado por la extensión de las libertades en general. El velo de ignorancia materializa su metodología, ya que permite pensar el desinterés de los ciudadanos como tales.

\section{II.2. EL ÍNDICE DE LOS BIENES BÁSICOS, INSTRUMENTO DE DEFINICIÓN DE LOS MÁS DESAVENTAJADOS}

Con el fin de distinguir los más desaventajados de los demás ciudadanos, la teoría de la justicia crea una base de información cuya validez depende de los criterios de decisión en vigor en el posicionamiento original. Así, estará exenta de todo lo que el velo de ignorancia disimula, a saber las informaciones particulares sobre los individuos. Por consiguiente, los socios del posicionamiento original hacen abstracción de las características particulares de las expectativas de los 
diferentes miembros de la sociedad, para retener únicamente las del ciudadano en general. «Puesto que los socios no tienen información precisa sobre ellos mismos o su situación, no pueden, de todas formas, identificarse» (Rawls 1971, p. 164). La elaboración de los principios de justicia no puede hacer un llamamiento a ninguna información particular o singular que se refiera a los nombres propios o a la noción de «descripciones definidas» puestas de manifiesto por Bertrand Russell. Por lo tanto, la búsqueda del interés individual se descarta del posicionamiento original, ya que el velo de ignorancia es el garante de la moralidad pura de los actores.

Rawls distingue una base de información general de una base de información universal. Una base de información que se refiere a una clase determinada de individuos, tal como la de los «ciudadanos en posesión de sus plenas facultades intelectuales y morales», es general. Una base de información es universal si se refiere a un nombre propio o a una descripción definida. Rawls ilustra ese caso con el ejemplo de la dictadura personal. Lo que es deseable para una persona racional está definido a partir de esta base de información general. Un lote de bienes, denominados «bienes básicos» es un lote de bienes susceptible de ser útiles en general a cualquier proyecto de vida razonable, sabiendo que este término hace referencia a las obligaciones que provienen de la cooperación social. A esta caracterización relativamente imprecisa, la segunda edición de Teoría de la justicia añade una determinación relativa a los propios objetivos de la teoría de la justicia: los bienes básicos son aquellos que permiten al ciudadano ser autónomo a nivel moral, y, por lo tanto, ser capaz de tomar unas decisiones que satisfacen los intereses prioritarios de la estructura básica de la sociedad. Responden a las necesidades y no a las preferencias particulares. Rawls evita así cualquier determinación arbitraria de la caracterización de los bienes básicos.

El estudio del contenido del índice de los bienes básicos permite averiguar esta aserción: si está determinado de manera no-arbitraria, este índice solo debería variar en unas condiciones fijadas de antemano. Rawls incluye entre los bienes básicos, «los derechos, las libertades y las posibilidades ofrecidas al individuo, las rentas y la riqueza», así como «las bases sociales del respeto de sí mismo» (Rawls 1971, p. 93). El reparto de los bienes básicos depende de la estructura básica de las instituciones. Este criterio de definición complementaria permite igualmente evitar cierta arbitrariedad. Pero, implica también que una privación de salud o de inteligencia no está considerada como una caracterización pertinente de la pobreza en el contexto de la teoría de la justicia. Si el índice de los bienes básicos está determinado, no significa que esté fijado. Así, a lo largo de la teoría de la justicia, las bases sociales del respeto de sí mismo completan este indicio.

Musgrave (1974) pone de manifiesto el problema que plantea la productividad de aquellos que, por gusto, prefieren unas actividades de escaso rendimiento 
económico en el trabajo, lo que perjudica la justicia redistributiva. Rawls ha tomado en consideración esa crítica, planteando el problema en los siguientes términos: si ciertas personas prefieren hacer surf en Malibú en lugar de ejercer un empleo, a pesar de su capacidad para ganar un salario, la teoría de la justicia de 1975 los incluirá en la categoría de los mal parados. En ese sentido, la solución consistiría en integrar el tiempo de ocio en la lista de los bienes básicos (Rawls 1988). Este ejemplo ilustra la preocupación de Rawls por independizar el índice de los bienes básicos de cualquier deseo, gusto o preferencia. Pero, las necesidades fundamentales para la determinación de este indicio varían según los individuos. Asimismo, ante la ausencia de cualquier información sobre las circunstancias particulares de la sociedad a legislar, es difícil definir lo que corresponde al ocio y al trabajo: el surfista que participa en unos campeonatos del mundo y está remunerado para ello trabaja efectivamente, pero su actividad propiamente dicha no difiere de la del surfista aficionado. La posición original no ofrece ninguna definición del trabajo: ¿Se trata de una actividad coactiva? $\mathrm{O}$ ¿es cuestión de una actividad útil? En realidad, la definición del trabajo y del ocio que presupone Rawls es tributaria de su concepción particular de la sociedad justa, inspirada en nuestras sociedades modernas. En estas condiciones, ¿la experiencia cognitiva del posicionamiento original puede llegar a su fin?

\section{II.3. EL VELO DE IGNORANCIA Y EL USO DE LA INTUICIÓN}

Los socios del posicionamiento original solo disponen de informaciones generales, ignoran sus posiciones sociales respectivas, incluso si tienen un conocimiento general de la organización social. Por lo tanto, desconocen su clase social y las ventajas naturales de las que disponen, tales como la inteligencia o la fuerza física, así como «el contexto particular de su propia sociedad» (Rawls 1971: 168-169).

La extensión del velo de ignorancia está definida por los objetivos de imparcialidad y de racionalidad perseguidos. Un levantamiento progresivo de este velo es posible. La capacidad humana para hacer unas elecciones pertinentes desde el punto de vista del interés general está presupuesta por la teoría del velo de ignorancia. Efectivamente, la posición original no es histórica, de manera que tendríamos la tentación de creer que la elección del principio de diferencia está motivada por un razonamiento egoísta, según el cual conviene garantizarnos de que formaremos parte de los peor parados en la futura sociedad. El posicionamiento original del velo de ignorancia constituye una experiencia cognitiva para comprender la lógica del razonamiento propiamente político.

Así, la base de información está establecida. Pero, la construcción de un índice de bienes básicos está intrínsecamente vinculada a la identificación de los más desaventajados. No en vano, ¿Cómo proceder a esta identificación, si la 
experiencia de esta situación particular no es accesible? ¿El velo de ignorancia no nos hace correr el riesgo de perder de vista las características principales de la pobreza? ¿Cómo circunscribir la diversidad de las situaciones posibles en un índice único, sabiendo que no se puede esperar una teoría coherente y utilizable si debe tomarse en consideración semejante multiplicidad de posiciones? (Rawls 1971, p. 126).

Para resolver esta dificultad, Rawls elabora en la Teoría de la justicia la noción de «posición social pertinente». Así, entre todas las posiciones sociales posibles, estas últimas representan un aspecto de la estructura básica cuya toma en consideración contribuye a la elaboración de los dos principios de justicia. Gracias a estas posiciones, los socios del posicionamiento original se identifican momentáneamente ante los miembros de la sociedad real, como objetos de reflexión política y no como portadores de intereses y de deseos no pertinentes para una teoría de la justicia como equidad. Pero, si el carácter «coherente y utilizable» de la teoría justifica estos posicionamientos, el objetivo de la justicia como equidad corre el riesgo de escaparnos. Como lo subraya Philippe Van Parijs, desde el punto de vista de la justicia, la selección de las «posiciones sociales pertinentes» es arbitraria y torpe, puesto que selecciona unas posiciones sociales contingentes, a partir de unos criterios cuya pertinencia no es evidente desde el punto de vista de la exigencia de justicia (Van Parijs 2003, p. 214).

Esta dificultad reconocida por Rawls resulta de que la definición de los peor parados obedece a unas contingencias naturales (talento, don) y sociales (origen familiar) que al carácter normativo del índice de los bienes básicos tiene dificultades para aprehender. El argumento pragmático del acabamiento de las capacidades de los socios del posicionamiento original, no es suficiente en este caso, incluso si aparece en varias ocasiones en el texto. Rawls formula entonces unas hipótesis sobre las posiciones pertinentes de individuos más desaventajados, pero no se pronuncia sobre un procedimiento definitivo. Así, propone identificar una posición particular, aquí la del obrero no cualificado, y proceder a la construcción del índice de los bienes básicos con respecto a su posición. Posteriormente, sugiere definir los más desaventajados a partir de la renta. Estos dos enfoques de la pobreza, una cuantitativa y otra cualitativa, son complementarios, porque son unos instrumentos de trabajo y no unos principios.

Pero, si el criterio cuantitativo es insatisfactorio, el criterio cualitativo plantea nuevas cuestiones. En un primer momento, teniendo en cuenta el carácter restringido de la base de información disponible en el posicionamiento original, la determinación de un individuo representativo es necesariamente arbitraria. El desconocimiento del contexto histórico o cultural de aplicación de la teoría de la justicia constituye la razón principal. Lo que no significa que la justicia social deba desaparecer en la expectativa de una hipotética mejora de las condiciones 
de vida: la propia enunciación del segundo principio da cuenta de la existencia ineluctable de desigualdades, incluso en una sociedad justa.

\section{La CRítica de Amartya Sen de los bIEnES básicos}

A partir de 1981, Amartya Sen expresa su insatisfacción ante el índice rawlsiano de los bienes básicos, a partir de sus propios trabajos sobre la pobreza. En contra de las definiciones y medidas de la pobreza más extendidas, Sen sustituye la noción de capacidad (capability) a la medida de las desigualdades de renta.

\section{III.1. UNA BASE DE INFORMACIÓN INVALIDADA}

Sen presenta el problema de la definición de la pobreza bajo la forma de una parábola: Annapurna, una joven mujer preocupada por la justicia social, desea contratar a un jardinero. Tres candidatos se presentan, sabiendo que sus competencias y exigencias son similares: por lo tanto, el más necesitado será seleccionado. El primero, Dinu, tiene la renta más baja; el segundo, Bishanno, es el más desgraciado, tras un revés de fortuna, y no para de quejarse; el tercero, Rogini, la única mujer, no se queja nunca aunque sufra una grave enfermedad y el dinero del salario propuesto sea suficiente para financiar su tratamiento. Annapurna duda y no sabe a quién contratar (Sen 1999,pp. 79-80). Una parábola tiene una dimensión moral y no es un género frecuentemente utilizado en la literatura económica. No en vano, la cuestión de la definición de las desigualdades se encuentra precisamente en el cruce de las evoluciones del pensamiento ético y económico.

El objeto de ese cuento, no es una comparación entre ricos y pobres, sino entre los propios pobres: para Sen, se puede ser pobre de diferentes formas. La cuestión «¿quién sufre de privaciones? Se desplaza hacia otra pregunta: «¿De qué están privados los pobres?». El análisis cualitativo de las desigualdades permite tener en cuenta una doble diversidad: la heterogeneidad de las necesidades y de los gustos, por una parte, y la multiplicidad de los criterios de medida de la igualdad, por otra parte.

Annapurna se encuentra en una situación análoga a la de los socios del posicionamiento original, porque los criterios de elección obedecen a su interés personal, tales como el salario que propone o la satisfacción que retirará de su futuro empleado, están descartados. Su motivación es desinteresada y el procedimiento de elección escogido obedece a la moral, en la medida en que no es reducible al interés individual. Sen, a la imagen de Rawls, sitúa desde el inicio la cuestión de la definición de la pobreza a un nivel moral.

Annapurna decide, por lo tanto, seleccionar el más desaventajado: se refiere a la concepción general de la justicia tal como la define Rawls compensando 
prioritariamente las desigualdades más importantes. Pero, esta selección evoluciona con las informaciones que recibe Annapurna. La primera base de información para su elección es la renta: Dinu es «el más miserable». Este criterio cuantitativo hace referencia explícitamente a uno de los criterios escogidos por Rawls para definir los más desaventajados. Pero, hemos visto que la noción de «posición social pertinente» complejiza la situación: ¿jamás Rawls afirma que las desigualdades socioeconómicas son exclusivamente unas desigualdades de renta, es decir que la crítica de Sen se equivoca de objetivo?

La segunda base de información considerada por Annapurna concierne los efectos de la pobreza sobre nuestro bienestar. Bishanno, acostumbrado a un nivel de vida más confortable que sus competidores, está más afectado que los demás por su pobreza actual. Definir Bishanno como el más pobre de los tres, como consecuencia de su insatisfacción, es inscribirse en la perspectiva del utilitarismo y de la economía del bienestar. A este propósito, Rawls se opone a la evaluación por las utilidades: las condiciones de elaboración de los principios de justicia impiden a sus actores tener en cuenta sus consecuencias y efectos. La satisfacción no está considerada como una medida válida de la ventaja, en la medida en que contribuye a la autonomía del ciudadano. La teoría de la justicia solo autoriza un principio de eficacia con la única condición de que los dos principios de justicia sean satisfechas. No en vano, aparece que la base de información utilitarista no completa y puede incluso contradecir los principios de la justicia.

El tercer candidato, Rogini, permite a Sen introducir nuevas categorías informativas, descuidadas tanto por Rawls como por los utilitaristas. Rogini está enferma, pero su salario de jardinera sería suficiente para financiar su tratamiento. No en vano, no es la más pobre de los tres, aunque acumule varias privaciones, debidas a su renta, a su salud que afecta a su bienestar, a su sexo y a su edad, que le impiden disfrutar de la consideración social requerida para sentirse investida del derecho de exigir lo que se le debe. Pero, el cúmulo de desigualdades cuestiona la selección de un solo criterio pertinente de definición de la pobreza, y esta situación pone de manifiesto los problemas inherentes a la conversión de los recursos en funcionamientos o en elementos que contribuyen a la calidad de vida. Así, el valor de un bien o de una renta varía en función de los individuos: el salario propuesto por Annapurna, aunque idéntico sea cual sea el jardinero seleccionado, tiene un valor superior para Rogini. El caso de Rogini hace referencia al rechazo de Rawls de incluir la salud entre los bienes básicos garantizados por las instituciones justas. Sen añade que Rogini es feliz: por lo tanto no forma parte de los más desaventajados para los economistas del bienestar y el utilitarismo. Pero, la continuación de la parábola muestra la dificultad propia a estas teorías: la felicidad no es un estado mental exclusivamente derivado de una cierta situación económica. Nuestra capacidad a soportar el dolor 
y las dificultades resultan también de nuestra cultura, de nuestra educación y de nuestra elección. La utilidad representa así una base de información inexacta.

¿Qué lección se puede sacar de esta parábola? Parece ser que Sen ha querido mostrar el reto que representan los criterios de selección de la base de información para definir las desigualdades. ¿De qué manera una teoría de la justicia puede justificar la exclusión de ciertas informaciones, tales como el hecho de que se inculque a una niña que no debe jamás quejarse y tener ambición? Sen utiliza frecuentemente el ejemplo de los discapacitados (Rogini sufre de una enfermedad incurable) en su crítica del principio de diferencia. La crítica por implicación de los casos cuestiona la idea de posición social pertinente, que presupone una cierta homogeneidad de las necesidades, haciendo abstracción de las variaciones entre individuos, tales como la salud, la longevidad, el clima o las condiciones de trabajo. Sen reintroduce así la categoría aristotélica de calidad para pensar la necesidad que Rawls valora en función del criterio de autonomía del sujeto. Los individuos solo pueden ser considerados como responsables de variaciones de las necesidades que no dominan (Sen 1991, p. 207).

Para analizar la teoría de la justicia, Sen se interesa por las informaciones utilizadas en dos componentes distintos pero vinculados de estas teorías: la selección de rasgos individuales pertinentes y la elección de estructuras combinatorias. En esta parábola, los rasgos individuales pertinentes son la renta para Dinu, la tristeza para Bishanno y la enfermedad para Rogini. Estos rasgos pertinentes pueden ser clasificados ellos mismos en dos categorías: algunos son evaluados en función de las posibilidades que ofrecen. Es el caso de los bienes básicos de Rawls, pero también de los derechos en la perspectiva de Nozick (1977). Otros dependen del resultado, sea cual sea el procedimiento utilizado para conseguirlo.

Las estructuras combinatorias influyen también en la base de información. La estructura combinatoria más simple es la igualdad de derechos. Otra posibilidad consiste en maximizar una suma: el utilitarismo procede así. Rawls propone una combinación más sutil ya que establece unas prioridades lexicales así como la posibilidad de utilizar un máximo. La dificultad en estas elecciones metodológicas estriba en conjugar la atención de las libertades y la toma en consideración de sus consecuencias. Si Sen rechaza el utilitarismo en razón de su desinterés por la cuestión de las libertades, critica igualmente en Rawls la diferencia entre la significación concedida a la libertad en la teoría de la justicia como equidad, y el que representa realmente, sobre todo para los más pobres. Sen considera que la economía moderna ha sido empobrecida por su separación con la ética. No obstante, la diversidad de las respuestas a esta pregunta refleja la diversidad de las bases de información posibles para definir la pobreza. Por lo tanto: ¿Cómo pensar esta pluralidad y cómo representarla bajo la forma de 
una clasificación coherente, si es posible completa, y que sea operativa para una teoría de la justicia?

\section{III.2. LAS CAPACIDADES}

Sen hace referencia en varias ocasiones a su deuda hacia Aristóteles (2001) para la elaboración del concepto de capacidad (Sen 1992, p. 66). Aristóteles introduce dicha noción que significa «tarea», «obra» y más específicamente «función» en su definición de la naturaleza de la felicidad. Si todos somos capaces de identificar la felicidad, nos dice Aristóteles, no conocemos por ello su naturaleza. No en vano, es un reto fundamental en la medida en que la ciudad y la comunidad política aspiran a la felicidad: si los hombres se ponen de acuerdo para vivir juntos, es porque esta cuestión de la felicidad les interesa. Aristóteles se interesa, por lo tanto, por la experiencia común: ¿Cuándo se habla de bien y de bien conseguido efectivamente? Esta idea de realización está vinculada a la noción de «función»: tocar el violín constituye una función que puede ser realizada o no. Cada oficio, cada ocupación del ser humano tiene una función e incluso cada parte del cuerpo tiene una: por qué el ser humano como tal no tendría una función propia, se pregunta Aristóteles. Procede a una dicotomía de la noción de vida y excluye progresivamente la «vida de nutrición» y la «vida de crecimiento» y posteriormente la «vida sensible»: ninguna de ellas es propia al ser humano.

La «vida práctica de la parte racional del alma» designa la vida activa, en oposición a la vida contemplativa. En el seno de esta vida, Aristóteles distingue la aptitud ya determinada, sometida a la razón, y el ejercicio de esta aptitud de cara a la realización de la función. No obstante, el ejercicio es determinante para la felicidad. Efectivamente, la función propia que determina una vida exitosa o una vida realizada, es la «vida según el punto de vista del ejercicio». Si se enfrenta la noción de «función propia» con la de «bien», en el sentido de «bienes básicos», se observa que Aristóteles introduce una dimensión cualitativa en la noción de «tener». Así, el texto Categorías dedicada al «tener» muestra la amplitud de las acepciones de este término: pueden tenerse unos conocimientos, una gran estatura, un vestido, un anillo, unas manos y unos pies, una casa o una pareja. La categoría de posesión muestra la amplitud de todo lo que el ser humano puede estar desposeído, no solamente verbalmente sino también realmente.

Encontramos en este texto la pluralidad que hacía falta a la base informativa de Rawls. Si los bienes básicos constituyen lo que tengo cuando estoy bien parado, parecen pobres comparados con esta categoría del «tener». De hecho, solo recubren la noción de posesión, ya que los demás modos del tener están relegados por Rawls en el ámbito de las contingencias naturales y sociales que la teoría de la justicia no controla. Si está comprendida en función del tener, la 
privación que caracteriza la categoría de los más desaventajados puede ser de tipo cualitativo: un defecto de conocimiento es la consecuencia de una escasa educación y formación, tema fundamental en el tratamiento de las desigualdades. Pero, la privación puede concernir la cantidad. Así, se puede pensar en los efectos de la malnutrición sobre el peso de los niños cuando nacen y, por lo tanto, sobre su peso cuando serán adultos. En cuanto a la privación de «lo que rodea el cuerpo», evoca la falta de vestido así como la situación de los sin hogares. La privación de «lo que está en una parte del cuerpo» es menos significativo: pensemos sin embargo en la función del adorno en la integración social.

En cuanto a la privación de una parte del cuerpo, hace referencia naturalmente a la existencia de discapacidades. La idea de contingencia puede evocar el hambre e incluso la hambruna. La lista no está cerrada: la diversidad de la realidad excede el intento de sistematizar definitivamente las privaciones. Las demás categorías son unos objetos posibles del haber: se puede tener una disposición, que hace referencia a la calidad, al tamaño, que se refiere a la cantidad, a una parte del cuerpo, que está vinculada a la sustancia. A la luz de este texto, la concepción de la pobreza en términos de privación de rentas e incluso de bienes parece muy restrictiva. Efectivamente, a diferencia de Rawls, Aristóteles no jerarquiza los diferentes «haberes» y deja abierta la posibilidad de ampliar todavía más esta categoría.

Sen deducirá de estas lecturas y de su propia experiencia como economista especialista del desarrollo, la noción de «funcionamiento». Estudiando las hambrunas, realiza una constatación paradójica: las hambrunas, contrariamente a lo que podría pensarse, no acontecen necesariamente en periodo de penuria alimenticia. Sobre el modelo de los estudios de caso presentados en Powerty and Famines, observemos la hambruna de 2000-2001 en Afganistán, analizada por la FAO (2001). A primera vista, esta hambruna ha sido provocada por las sequías sucesivas de 1999, 2000 y 2001. La debilidad de las precipitaciones ha tenido un impacto sobre las cosechas no irrigadas, que han tomado una importancia creciente en razón del mal estado del sistema de irrigación tradicional que se ha visto afectado por los años de conflicto que ha vivido el país. En su conjunto, la producción de cereales afgana ha disminuido del 37\% entre 1999 y 2001.

Pero, un análisis profundizado pone de manifiesto unas interesantes disparidades regionales. Así, en el Este del país, la producción de trigo (la principal cereal cultivada y consumida en el país) ha aumentado, después de un año 2000 ciertamente difícil, pero que solo representa una disminución del 23\% con respecto a 1998, es decir mucho menos que en el resto del país. El incremento de la producción de trigo los demás años se debe al abandono de la cultura de la adormidera a opio a partir de 1999, siguiendo las órdenes de los Talibanes que gobernaban el país. Estas regiones habrían podido paliar este ligero déficit de la producción de trigo comprando unos cereales al Pakistán vecino. La FAO 
identifica los grupos sociales más vulnerables con el fin de definir la ayuda alimenticia que conviene aportarles. No en vano, en el Este, los grupos vulnerables son los «agricultores tributarios de la cosecha, incluso los cultivadores de adormidera y los obreros agrícolas». Así, la bajada del poder adquisitivo de los trabajadores agrícolas sin tierra y más especialmente de los trabajadores de la adormidera, no está vinculada a la disminución de los recursos alimenticios y a la sequía, sino a un fenómeno que no tiene nada que ver con una catástrofe natural: la erradicación de la cultura de la adormidera a opio. Incluso si la producción de cereales aumenta el año siguiente, estas personas vulnerables no podrán procurarse comida sobre el mercado por falta de recursos financieros.

La pérdida de los ingresos de la adormidera a opio para el gobierno talibán ha tenido un impacto notable sobre el acceso a la comida para la población: privada de recursos fiscales, las autoridades no han podido importar unas cereales con el fin de venderlos a unos precios subvencionados así como lo habían hecho el año anterior. Asimismo, las negocios que vivían de una manera u otra de la cultura de la adormidera y que habrían podido importar trigo con unos fines comerciales no lo han hecho, participando así al déficit alimenticio.

Así, resumir los factores de la hambruna de 2000-2001 en Afganistán a la sequía supone descuidar la diversidad de las situaciones y de las capacidades de enfrentarse a un cambio forzado de modo de vida para cada grupo social. En su conjunto, los grupos más vulnerables son aquellos que dependen exclusivamente de un modo de acceso a los recursos alimenticios, sea cual sea el estado de estas últimas: los pequeños aparceros, que solo dependen de la producción pluvial; los nómadas, que dependen únicamente de la ganadería; los hogares cuyo jefe de familia es una mujer, y que no tienen acceso al mercado laboral y dependen de la caridad; los trabajadores ocasionales, como los trabajadores de la adormidera. El análisis de esta hambruna se ha desplazado por lo tanto de la disponibilidad alimenticia hacia el acceso a estos recursos. Así, Sen ha puesto de manifestó la importancia de disponer de posibilidades alternativas para acceder a la comida y resistir a los fenómenos de hambruna. En este sentido, propone dibujar una cartografía de los derechos de acceso, que definiría mejor la realidad de la privación que la simple descripción cuantitativa de las rentas y de los recursos disponibles.

La capacidad es el concepto producido por dicha cartografía y designa la ordenación de los posibles funcionamientos para realizar un cierto proyecto de vida (Sen, 1992). Designando la posibilidad de funcionamientos múltiples en lugar de la realidad de un funcionamiento particular, Sen puede decir que su actor económico es libre, una libertad que se refiere a la calidad de vida. Sen transpone así un método descriptivo de los fenómenos económicos a un espacio que pertenece a la ética, el espacio de lo que constituye precisamente la felicidad. 


\section{CONCLUSIÓN}

El análisis comparativo de los escritos de Rawls y de Sen sobre la pobreza ha hecho aparecer la garantía de la especificidad de lo político como un reto fundamental para la consistencia de la teoría de la justicia como equidad. Si la primera respuesta de Rawls a Sen parece errónea porque no ha percibido el aspecto metodológico y epistemológico de la crítica de los bienes básicos, hace resaltar la razón fundamental de esta elección metodológica: la garantía del ejercicio de la ciudadanía, identificada con una motivación política pura, motivación moral en el sentido de que es autónoma y tiene como finalidad el interés general. El problema de las condiciones de ejercicio de la libertad provoca, por lo tanto, una reflexión sobre la racionalidad de los actores políticos, con el fin de determinar si la autonomía puede caracterizar esta racionalidad y si la concepción de la persona según Rawls puede legítimamente fundar la teoría de la justicia.

No en vano, aparece que no se puede postular el carácter completo a priori de la teoría de la justicia. La realidad excede y a veces invalida la base de información disponible para definir la pobreza. Esa realidad excesiva ofrece la materia de la crítica por la implicación de casos de la teoría de la justicia como equidad, y especialmente de la prioridad del principio de libertades iguales para todos sobre el segundo principio, que incluye el principio de igualdad de oportunidades y el principio de diferencia. ¿Es decir que la teoría de la justicia no puede alcanzar el nivel de abstracción requerido por un pensamiento de tipo contractualista? En realidad, poniendo el énfasis en el aspecto relacional que caracteriza los funcionamientos y la cartografía de los derechos de acceso a los recursos, Sen demuestra un nivel de abstracción superior al de la teoría de la justicia. Efectivamente, el fracaso parcial de la sociedad bien ordenada de la Teoría de la justicia, fracaso que se traduce por un viraje en Liberalismo político (1993) donde Rawls restringe la aplicación de su teoría a las «democracias occidentales contemporáneas», parece confirmar nuestra crítica de la presencia de entidades metafísicas en el seno de la teoría que pretende ser exclusivamente política.

Los bienes básicos no están vinculados a una concepción homogénea de la persona que se fundamenta inicialmente en unos datos psicológicos y posteriormente en unos elementos históricos y culturales, como la Constitución americana o la Reforma. Esta concepción que excluye los defectos de la racionalidad pone en peligro la garantía del bien básico más importante, las bases sociales del respeto de sí mismo; bien básico que paradójicamente legitima la prioridad del primer principio pero cuya garantía depende del segundo principio. Por «defecto de racionalidad», designamos la perturbación de la formación de las preferencias, los conflictos de cooperación en el seno de la familia, y el conjunto 
de los factores que perjudican la percepción de sí mismo como un ciudadano cualquiera, teniendo razón a la hora de elegir efectivamente. Los ejemplos que expone Sen conciernen con frecuencia a las mujeres. No solamente las mujeres son solamente víctimas de desigualdades en las democracias occidentales contemporáneas concernidas por la teoría rawlsiana, sino que además, la justicia no puede restringir su ámbito de aplicación según unos criterios económicos o geográficos.

Afirmando que la crítica de Sen conduce la teoría de la justicia hacia un nivel de abstracción superior, queremos decir que el cuestionamiento del índice de los bienes básicos corresponde a un análisis crítico del postulado de la concepción de la persona. ¿Este postulado constituye un requisito? El enfoque por las capacidades y la crítica de la homogeneidad de los bienes básicos han eliminado todas las entidades que engloban la teoría de la justicia. Dicha teoría puede ser considerada como un caso particular de la justicia en general. Pero, esta concepción más general de la justicia se olvida de postulados tales como la concepción de la persona, la distinción de lo político y de lo metafísico o la prioridad del primer principio.

Sin embargo, esta crítica ha hecho aparecer las dificultades inherentes a la nueva concepción de las libertades, concebidas como unas «libertades instrumentales». Efectivamente, los criterios que permitirían legitimar unos principios, reglamentos y legislaciones futuras han sido descartados, haciendo correr el riesgo de hacer caer esta nueva perspectiva en un relativismo insatisfactorio. La solución preconizada consiste, por lo tanto, en hacer de la teoría de la justicia un sistema incompleto. Sean cuan sean los posibles desacuerdos entre los diferentes actores de la decisión política, no es necesario resolverlos en su conjunto. La toma en consideración de las consecuencias y de los resultados, que solo es posible porque el sistema es incompleto, solamente permite tomar en consideración las preferencias requeridas para la solución. Un desacuerdo importa poco si no está vinculado a un objetivo concreto actualmente perseguido.

Para la epistemología de las ciencias humanas, la aportación de la crítica de los bienes básicos por Sen es haber integrado una dimensión cualitativa al trabajo de definición de la pobreza, con un índice de desarrollo humano que se ha convertido en un criterio seleccionado por las organizaciones internacionales. La sustitución de las capacidades a la renta ha puesto de manifiesto el presupuesto fetichista de las políticas de justicia redistributiva, proponiendo interesarse por el uso y la conversión de los recursos en funcionamientos o en libertades efectivas, en lugar de la disponibilidad o del valor absoluto de los recursos.

Desde el punto de vista ético, Sen ha confortado la exigencia moral de la teoría de la justicia, que implica una cierta concepción de la persona. Estudiando las condiciones de realización de los derechos, Sen no cuestiona estos últimos, 
sino que propone reforzarlos convirtiéndolos en concebibles para unos individuos variados. El objetivo consiste en relativizar la concepción homogénea y quizás arbitraria del actor racional sin por ello caer en el relativismo. El enfoque de Amartya Sen conduce a una concepción abierta de lo político que puede, en función de las consecuencias, integrar unos datos que Rawls había clasificado como no-políticos. Es en esta óptica que Sen propone fiarse al debate y a las elecciones democráticas, a los que se concede cierta libertad gracias a la apertura y al carácter incompleto del sistema de elección colectiva.

\section{REFERENCIAS BIBLIOGRÁFICAS}

ARISTÓTELES, 2001: Ética a Nicómaco. Madrid: Alianza Editorial.

FAO (Food and Agriculture Organisation), Mission FAO/PAM d'évaluation des récoltes et des disponibilités alimentaires en Afghanistan, 8 juin 2001.

KANT, E. 2000: Crítica de la razón práctica. Madrid: Alianza Editorial.

MUSGRAVE, R. 1974: «Maximin, uncertainty, and the leisure trade-off», Quarterly Journal of Economics, $\mathrm{n}^{\circ} 88$.

NOZICK, R. 1977: Anarchy, State and Utopia. New York: Basic Books.

RAWLS, J. 1971: A Theory of Justice. The Belknap Press of Harvard University Press.

RAWLS, J. 1988: « The priority of right and ideas of the good», Philosophy and Public Affairs, vol.17, pp.251-276.

RAWLS, J. 1993: Political Liberalism. New York: Columbia University Press.

SEN, A. 1991: On Ethics and Economics. Oxford: Blackwell Publishers.

SEN, A. 1992: Inequality Reexamined. Oxford: Oxford University Press.

SEN, A. 1999: Development as Freedom. New York: Alfred A. Knopt Inc.

VAN PARIJS, P. 2003: « Difference principles », in The Cambridge Companion to Rawls. Cambridge: Cambridge University Press.

EGUZKI URTEAGA es Profesor de Sociología en la Universidad del País Vasco e Investigador en el Centro de investigación IKER, laboratorio asociado al CNRS francés.

Línea de investigación:

Director del Grupo de Investigación de Estudios Vascos

Publicaciones recientes:

(2007) La politique d'immigration du gouvernement basque, Paris: L'Harmattan.

(2008) Les Plans Locaux d'Immigration en Espagne, Paris: L'Harmattan.

Dirección electrónica: eguzki.urteaga@ehu.es 